Dra. Carmen CAFFAREL-SERRA

Universidad Rey Juan Carlos. España. carmen.caffarel@urjc.es

\author{
Dr. Mario GARCÍA-DE-CASTRO \\ Universidad Rey Juan Carlos. España. mario.decastro@urjc.es
}

\title{
Futuro de la televisión pública nacional y autonómica en España. Propuestas de los partidos políticos en las elecciones de 2015 y 2016
}

\author{
The Future national and regional public television in Spain. Proposals of Political Parties in 2015 \\ and 2016 Elections
}

Fechas | Recepción: 03/10/2016 - Revisión: 31/10/2016 - Publicación final: 01/01/2017

\begin{abstract}
Resumen
La segmentación del panorama político español y la desaparición de las mayorías absolutas ha propiciado el protagonismo del debate sobre el servicio público de la televisión. Este trabajo presenta los resultados de la investigación de las posiciones sobre la televisión pública en España expresada por los principales partidos políticos que han concurrido a las convocatorias electorales celebradas en España durante 2015 y 2016. Hemos partido de la hipótesis de que la fortaleza y viabilidad del servicio público de televisión es ahora mayor que en 2012 cuando existían mayorías absolutas en el parlamento nacional y en varias de las comunidades autónomas. Metodológicamente hemos realizado un análisis de contenido de las propuestas sobre la televisión pública nacional y autonómica a partir de los programas electorales de los partidos políticos para las elecciones autonómicas de 2015 y generales de 2015-2016 y en paralelo hemos realizado entrevistas estructuradas con los responsables de comunicación de los cuatro partidos mas representativos a nivel nacional. A partir del análisis hemos comprobado que las televisiones públicas en España tienen mas futuro y mas viabilidad con la fragmentación del nuevo escenario político que cuando existía sólo la alternancia PP, PSOE.
\end{abstract}

\section{Palabras clave}

Consejo audiovisual; Estado; financiación; medios públicos; partidos políticos; televisión pública

\begin{abstract}
The scattering of the Spanish political landscape and the disappearance of absolute majorities have led to the prominence of debate on television public service. This paper presents the research results of the positions on the public media in Spain, expressed by the main political parties that have attended election calls held here during 2015 and 2016. We have started from the hypothesis that strength and viability of television public service is now bigger than in 2012 when there were absolute majorities in the National Parliament and in several autonomous communities.Methodologically speaking, we have conducted a content analysis of the proposals on national and regional public television from the electoral programs of political parties for 2015 regional and 2015-2016 general elections. In parallel, we have conducted structured interviews with those responsible for media and communication of the four most representative parties at national level. From analysis we have confirmed the hypothesis that public televisions in Spain have more future and viability with the fragmentation of the new political landscape.
\end{abstract}

\section{Keywords}

Audiovisual Council; State; Financing; Public Media; Political parties; Public television 


\section{Introducción}

Aunque las posiciones de los partidos políticos respecto a los diferentes vectores de actuación pública suelen diferir cuando están en el gobierno o cuando ocupan la oposición, son estas fuerzas políticas las que deben de resumir las aspiraciones y perspectivas sociales en torno a los diferentes aspectos que determinan el futuro de un país. El futuro de los medios de comunicación públicos depende de la voluntad y confianza de los ciudadanos respecto a su utilidad social y por consiguiente de la voluntad reguladora de la administración pública. La administración pública está regida por el aparato de gobierno y gestión de los intereses públicos, conformado por las fuerzas políticas con representación parlamentaria mayoritaria. Son las posiciones de esos partidos políticos con representación parlamentaria, expresadas en sus programas y proyectos legislativos, las que pueden determinar y marcar el futuro de nuestro objeto de estudio, el servicio público de televisión.

Para conocer el futuro de los medios públicos de comunicación en España hemos analizado las propuestas y posiciones de los diferentes partidos políticos con intención de gobierno en el escenario electoral de los últimos años. La gran novedad es que el escenario político español ha cambiado pasando de un sistema bipartidista o gubernamental a un sistema multipartidista o parlamentario de cuatro formaciones principales, con el nacimiento de nuevos partidos políticos denominados emergentes. Esta evolución parece haber devuelto protagonismo al papel de los medios públicos.

Es cierto que en los últimos años se había producido una fuerte tendencia social a favor de la televisión privada, también es cierto que, desde el año 2011, y como consecuencia de los movimientos sociales y las nuevas formaciones políticas emergentes, la radiotelevisión pública, junto la sanidad, la educación o la cultura, ha vuelto a adquirir la importancia social perdida en España y el escenario político español se ha reconfigurado reclamando un debate sobre su modelo.

Hay mucha literatura sobre este tema, análisis de los cambios legales a partir de la Ley 17/2006 (Azurmendi, López \& Manfredi, 2011; Zallo, 2010), los modelos resultantes (Caffarel \& García de Castro, 2006; López Cepeda, 2012; López Jordán, 2013), la financiación del servicio público (Bustamante, 2013a; De Mateo \& Bergés, 2009; Ortiz, 2010), comparación de la situación económica- financiera de la radiotelevisión pública en España con otros ejemplos de gestión de países como Francia, Reino Unido y Alemania (Jivkova, 201 1); la regresión del pluralismo en los medios (Bustamante, 2013b); la precarización del sistema de trabajo del creador audiovisual en el sector público CRTVE por la falta de iniciativas de financiación y segmentación de audiencias (Heredero \& Reyes, 2016), el futuro de las televisiones autonómicas (Bustamante, 2010; FORTA, 2012; Marzal, 2015; Miguel \& Casado, 2015, López-Rico, 2016 ) o el futuro del servicio público en Europa (Lowe \& Bardoel, 2007; Moreno \& Jiménez, 2007, Campos-Freire 2013). Sin embargo, falta una aproximación a la realidad de su futuro, basada en la opinión pública y publicada de los partidos políticos en los comicios autonómicos y generales de 2015 y 2016, en los que la novedad es la irrupción de nuevos partidos que han colocado en la agenda pública el debate sobre el futuro de la televisión pública arrastrando, en alguna medida, a los partidos llamados tradicionales.

\section{Contexto político español}

La crisis económica y los recortes sociales han tenido como consecuencia profundos cambios en el escenario de los medios de comunicación, con el desplazamiento del interés del público hacia otros medios o los procesos de reforma y contrarreformas por parte de las administraciones públicas correspondientes (europea, estatal, autonómica), que han traído como consecuencia la desaparición o la privatización de medios.

En España, la Ley 17/2006 de 5 de junio, de la radio y la televisión de titularidad estatal, refundaba una nueva Corporación de Radio y Televisión Pública (CRTVE) basada en la elección de su presidente por mayoría reforzada de dos tercios de la cámara de diputados y con una gestión de seis años de mandato para que no coincidiera con las legislaturas. De igual modo, la ley 8/2009 de 28 de agosto, perseguía la estabilidad financiera y la independencia económica de la nueva Corporación, una financiación mixta al $50 \%$ entre un canon anual de las televisiones comerciales y las operadoras de telecomunicaciones, y el resto con los fondos de los Presupuestos Generales del Estado. Estas leyes pretendían marcar el inicio de una nueva etapa para sentar las bases de una radiotelevisión pública. A partir de entonces se crearon los Consejos de Informativos, redactaron el Estatuto de Informativos de TVE, y aparecieron nuevos programas de debate e información pública en la programación. Del 2007 al 2011 los dos presidentes de la nueva CRTVE fueron elegidos por consenso entre los partidos mayoritarios de las Cámaras.

Con posterioridad, y tras las elecciones generales del 2011 y el cambio de gobierno del Partido Popular, se inició un deterioro de la calidad del modelo de servicio público: el Real decreto- ley 15/2012 de 20 de 
abril modificó el régimen administrativo de la CRTVE, y el nuevo Presidente fue elegido sólo por mayoría absoluta de la Cámara, transcurridas tan sólo 24 horas de la primera votación. Y por otro la creación del Consejo Estatal de Medios Audiovisuales (CEMA) que estaba prevista en la Ley General de Comunicación Audiovisual del 2010, fue desestimada a partir de la llegada al poder del Partido Popular quien integró sus funciones en la Comisión Nacional de los Mercados y la Competencia (CNMC) (Gavara, 2013).

En el año 2008 se inicia una crisis económica de alcance mundial que afecta también a los presupuestos de los entes públicos audiovisuales, crisis que acabará dando lugar a la Ley de Estabilidad Presupuestaria y Estabilidad Financiera, de mayo 2012. Por otra parte la modificación de la Ley General de Comunicación Audiovisual de agosto de 2012, abre la puerta a la posible privatización de las televisiones autonómicas. Las consecuencias en los dos años siguientes fueron los expedientes de regulación de empleo en la Radiotelevisión valenciana (RTVV) con el cierre de la cadena a continuación; también en Telemadrid y en la radiotelevisión murciana (RTVM). Con despidos masivos de más del $70 \%$ de las plantillas, y reducciones presupuestarias en todas las radiotelevisiones públicas, tanto en la estatal como en las autonómicas. Todos estos cambios de escenario político y la inestabilidad institucional contribuyen desde entonces en nuestro país a la quiebra de la confianza ciudadana en la necesidad del servicio público de la radiotelevisión. Una confianza que claramente estuvo determinando su futuro.

Las funciones del servicio de radiotelevisión pública están en crisis en Europa desde hace ya varios lustros, y mientras parece ganar terreno hegemónico en los sistemas mediáticos europeos el modelo americano, a la vez que se registra una mayor tendencia al oligopolio de los grandes grupos privados. En Europa, y por ende también en España, hemos asistido a un proceso progresivo de desregulación del servicio público y de pérdida de identidad de sus funciones (Moreno \& Jiménez, 2007). La crisis económica reciente ha conllevado recortes muy profundos en los recursos de los medios públicos, como por ejemplo con el cierre de la televisión griega (ERT) el 11 de junio de 2013, o en el caso del ámbito regional español con el cierre de la RTVV, que fue clausurada en la madrugada del 23 de noviembre de 2013.

Por otra parte, la despolitización en su funcionamiento y la profesionalización en el desarrollo de sus actividades y misiones ha sido un objetivo de principio de las radiotelevisiones públicas europeas más asentadas. Tanto de la británica BBC desde su fundación, como el de otros medios públicos de los países nórdicos o escandinavos de Europa. Mientras que, en el caso español, o el del sur de Europa, a pesar de los procesos de reforma y contrarreforma del marco jurídico y administrativo, la autonomía jurídica en la gestión del servicio público de radiotelevisión sigue pendiente (García de Castro, 2006). Parece lógico, por tanto, que a día de hoy su autoridad y su futuro dependa del interés colectivo de su actividad, y de la ineludible autonomía e independencia editorial, administrativa y económica.

Estas políticas públicas de comunicación han sido y son objeto de diferentes investigaciones a lo largo de la historia, ello ha tenido como consecuencia que se pueda hablar de diferentes modelos, puesto que en la mayoría de los países las autoridades reguladoras suelen seguir unas pautas bastante similares. Según Hallin y Mancini (2004) el modelo español (Modelo pluralista polarizado) está determinado por el sistema electoral mayoritario, en el que la radiotelevisión pública estaría controlada por el gobierno de turno. Ahora el contexto político esta evolucionado del bipartidismo imperfecto al multipartidismo proporcional de la política de consenso, en el que prima el reparto de poder. Y es en estos modelos donde frente a la instrumentalización política se afianza la profesionalización del servicio público.

Aunque como también sostienen Hallin y Mancini (2004: 49) con el tiempo el sistema gubernamental acaba adoptando el modelo profesional-liberal, ya que las alternancias en el poder hacen que las fuerzas políticas acaben aceptando la pérdida de control y prefieran no pasar éste a sus rivales lo cierto es que ahora todos los partidos, como podrá verse a continuación, se pronuncian a favor de la profesionalización y la independencia en la regulación de la radiotelevisión pública.

\section{Objetivos}

Esta aportación presenta los resultados del trabajo de investigación y análisis de las posiciones sobre los medios de comunicación públicos en España expresadas por los principales partidos políticos que han concurrido a las convocatorias electorales celebradas en España durante 2015 y 2016. Para poder sintetizar en este artículo los resultados de nuestra investigación hemos establecido cinco grandes calas en la extensa doctrina recogida durante el trabajo de campo realizado, que se detalla a continuación. A partir de estos cinco grandes ítems hemos tratado de jerarquizar las propuestas de los diferentes partidos que han concurrido a las convocatorias electorales, así como la exposición de nuestras conclusiones:

1. El servicio público de radiotelevisión.

2. Coexistencia entre la radiotelevisión pública nacional y las autonómicas. 
3. Gobernanza y la transparencia.

4. Financiación y sostenibilidad del modelo de televisión pública.

5. El Consejo Audiovisual.

De este modo, los objetivos de esta investigación son los de conocer el futuro del sistema mediático de la radiotelevisión pública en España según el contexto político actual. Y de este modo contribuir a determinar la función social de los medios de comunicación en España a través de las políticas públicas propuestas por los diferentes partidos políticos que han concurrido a las últimas elecciones (municipales, autonómicas y generales)

Acabamos de vivir dos años de gran intensidad de la actividad electoral de los partidos políticos, tanto tradicionales como emergentes. Dos años, 2015 y 2016, en los que se han celebrado elecciones autonómicas y municipales y dos convocatorias de elecciones generales, en las que se han producido novedades con los programas electorales de los llamados partidos "emergentes" o nuevas formaciones políticas, que han arrastrado a un posicionamiento del resto.

Partimos de la hipótesis de que, con todas estas novedades en el escenario mediático, y la segmentación del electorado en las diferentes opciones políticas que han concurrido a las elecciones, las distintas fuerzas políticas han debido definirse y concretar sus propuestas respecto al futuro de los medios públicos. Ello nos permitirá indagar sobre el futuro y la mayor o menor salud del sistema público de medios de comunicación de nuestro país, a la luz de las posiciones de los diferentes partidos políticos que ocuparan el gobierno de la administración pública. Así como concretar las posibles funciones sociales que van a cumplir los medios de comunicación en el futuro de nuestro país.

De estos objetivos ha surgido una hipótesis que tratamos de confirmar o refutar en nuestras conclusiones: ¿tiene hoy mejor o peor salud el sistema público de radiotelevisión en España que en los últimos años? ¿̇Tiene mayor o menor futuro?

\section{Metodología}

Para conocer ese futuro diseñamos un proyecto de investigación con dos etapas diferenciadas, aunque complementarias: la primera de ellas abarca el periodo electoral que se inicia con las elecciones autonómicas en Andalucía el 22 de marzo de 2015, la convocatoria de las elecciones autonómicas y municipales del 24 de mayo de 2015 y culmina con las elecciones catalanas del 27 de septiembre del 2015. La segunda comprende desde las elecciones generales celebradas el 20 de diciembre del mismo año hasta la nueva convocatoria de elecciones generales de junio del 2016 ii.

Para cada fase del estudio y cada convocatoria electoral autonómica y general, se procedió a recoger como fuentes primarias los programas de los diferentes partidos políticos que concurrían a las elecciones, diferenciándolos por comunidades autónomas en las que había proceso electoraliii. Es cierto que los programas electorales por autonomías no difieren sustantivamente del programa marco del propio partido en cuestión elaborada para esas convocatorias, pero no es menos cierto que presentan algunas diferencias que señalamos y sobre todo buscamos las causas de estas diferencias para compararlas con los programas marco de los diferentes partidos políticos o coaliciones en las elecciones autonómicas y municipales. Éramos conscientes de que en aquellas Comunidades Autónomas en las que el servicio de televisión pública se había clausurado (Valencia) o jibarizado (Madrid), los programas de los diferentes partidos en esa Comunidad iban a hacer mayor hincapié que en los programas marcos. Lo mismo sucedía en aquellas comunidades autónomas en la que ya existía un Consejo Audiovisual como por ejemplo el caso de Cataluña o Andalucía.

Para el análisis de los datos se excluyó a aquellas comunidades cuya radiotelevisión estaba encomendada a la gestión privada (Castilla, La Rioja y Navarra), o el caso de Cantabria, que no tiene. En esta primera fase del trabajo se tuvieron por tanto en cuenta sólo los programas marco de las elecciones autonómicas y los particulares para cada Comunidad Autónoma.

Después de recoger y clasificar todos los programas -y tras el resultado electoral- se analizaron solo los programas de aquellos partidos que ocuparon las primeras posiciones en número de escaños a nivel nacional (Ciudadanos, Podemos, Partido Popular, Partido Socialista Obrero Español)iv. Por supuesto se incluyó en nuestro análisis los programas de las plataformas y coaliciones que han tenido un papel destacado en el resultado electoral, como el caso de Junts pel Si para Cataluña o Compromís para la Comunidad Valenciana. 
En la segunda fase del trabajo de investigación, se analizaron los programas electorales generales en las dos convocatorias del 20 de diciembre de 2015 y de 26 de junio de 2016 , aunque no hubiera diferencias sustanciales entre ellos. Luego, para el desarrollo del trabajo, sólo hemos tenido en cuenta los programas electorales de los principales partidos de ámbito nacional: Ciudadanos, Podemos, PP y PSOE, con lo que hemos podido comparar sus propuestas en el ámbito autonómico y el nacional.

En la primera fase de la investigación se tomaron también en cuenta los discursos de investidura de los nuevos presidentes autonómicos como la segunda fuente primaria utilizada. Ya que en mas de un caso, el candidato a presidente hizo referencia al servicio público de radiotelevisión de su comunidad autónoma como por ejemplo el caso de Ximo Puig en la Comunidad Valenciana.

Una vez realizado el análisis de contenido de los programas electorales y de los discursos de investidura se decidió, a fin de obtener datos cualitativos que nos permitieran entender con mayor rigor algunas de las afirmaciones que aparecían en los programas, hacer entrevistas estructuradas. Se opto por una muestra de conveniencia ya que sólo se entrevistaron a los responsables de comunicación (o en quienes delegaron) de los cuatro partidos mayoritarios en los resultados de las elecciones generales. En las elecciones de diciembre de 2015 el cómputo de escaños del PP, PSOE, Podemos y Ciudadanos representó el $88,7 \%$ del total de diputados. Las entrevistas fueron grabadas y posteriormente transcritas para su análisis.

La entrevista estructurada, igual para todos los partidos, tenía una primera parte dedicada a las televisiones autonómicas (modelo, gobernanza, funciones, contenidos) una segunda igual, pero a nivel nacional y un tercer apartado en el que se preguntaba por la coexistencia de ambos sistemas, al constituir una particularidad del servicio público de radiotelevisión de nuestro país` (Caffarel 2007)

Con los resultados obtenidos hemos tratado de desarrollar una valoración prospectiva del papel social de los medios públicos en España. Por un lado, los análisis de contenido sobre los programas electorales nos proporcionaron datos para contrastar las diferentes propuestas de los partidos. Paralelamente, los datos cualitativos del trabajo nos los proporcionaron las entrevistas que se realizaron a los partidos que concurrieron a las elecciones generales. Por último, hemos procedido al análisis de las notas de prensa, que abordan el tema de los medios de comunicación, emitidas por los cuatro partidos analizados y que están publicadas en sus respectivas páginas webs, en el mismo periodo electoral. A su vez el seguimiento de medios de comunicación durante las campañas electorales nos proporciona datos secundarios muy útiles para conocer las diferentes propuestas y el comentario que suscitan en los distintos medios teniendo en cuenta las firmas y la cabecera del mediovi.

\section{Análisis de los datos}

\subsection{El servicio público de radiotelevisión}

Todos los partidos políticos, excepto el PP, explicitan en sus programas electorales la importancia de los medios públicos para la construcción de una sociedad avanzada, e incluso varios de ellos les dedican un capítulo específico. El PSOE, para las generales de 2015, "Una visión progresista de la sociedad de la comunicación"vii El programa de Podemos, en Comú Podem y En Marea aborda el tema en "RTVE y políticas mediáticas"viii Ciudadanos aborda su propuesta sobre los medios públicos en el epígrafe "La cultura, columna vertebral de un Paísix.

El PP, en los programas autonómicos para Andalucía, Cataluña y Canarias, sí habla del servicio público de radio televisión y a veces de forma contradictoria: en el caso de Andalucía dice textualmente: "Apoyaremos a la RTVA como medio de comunicación social de carácter público y aplicaremos una gestión que hagan de esta empresa un verdadero servicio público eficiente e independiente, aprovechando al máximo los recursos económicos, materiales y personales de esta Agencia pública"x.

Sin embargo, en Canarias propone literalmente "privatizar" el medio "Diseñaremos y llevaremos a efecto el procedimiento de licitación, en orden a su privatización, del Ente Público Radiotelevisión Canaria, salvaguardando los contratos de los trabajadores actualmente en vigor, y destinar los fondos públicos liberados a políticas sociales y fomento del empleo"xi.

Cabe mencionar que en estas comunidades el gobierno está sustentado por otro partido diferente al PP. Por tanto, el PP tiene una posición más variada, por no decir contradictoria, respecto a las radiotelevisiones autonómicas, ya que en unos casos apoya su carácter público (Andalucía) y en otros se muestra partidario de su privatización (Canarias). En nuestra entrevista, este partido contestó posicionándose claramente a favor de la titularidad pública de la radiotelevisión nacional y de las autonómicas, aunque 
especificó a continuación que "existen fórmulas en algunas autonomías de titularidad pública y gestión privada muy útiles"(Entrevista PP)xii.

Para el resto de partidos, las referencias de los programas electorales autonómicos y de los programas marco de Ciudadanos, PSOE y Podemos abogan unánimemente por una decidida apuesta por el mantenimiento del servicio público de televisión que garantice su futuro y para ello proponen diferentes alternativas:

Ciudadanos, en la mayor parte de programas electorales autonómicos, apoya la autonomía e imparcialidad de los entes públicos autonómicos y se pronuncia por la despolitización y la regeneración democrática, por su mejora y perfeccionamiento a través del nombramiento de sus cargos por consenso y mayoría reforzada, posicionándose en la línea de la reforma establecida por la ley derogada del anterior gobierno de Zapatero. A modo de ejemplo en el programa de Castilla-La Mancha dicen literalmente:

Ciudadanos modificará la actual regulación de la radio y televisión pública para dotarla de imparcialidad. De modo que el consejo de administración y el director general sean nombrados por mérito y capacidad y por mayoría de las tres quintas partes del Pleno de las Cortes, dificultando así que un solo grupo parlamentario pueda realizar estos nombramientos (2015:54)xiii.

Se pronuncia también a favor de recuperar la RTVV en la Comunidad Valenciana y despolitizar y profesionalizar Telemadrid. En el programa de Aragón aboga por "garantizar la independencia y pluralidad de los medios públicos de titularidad autonómica" y en Baleares por la regeneración democrática contra la colonización de los medios.

Observamos una correspondencia importante entre estas afirmaciones recogidas en los programas electorales y el pronunciamiento que hacen en sus discursos los candidatos a las presidencia de las comunidades autónomas como podemos observar en el siguiente cuadro:

\section{Cuadro 1: Referencias al servicio público de radiotelevisión en los discursos de investidura a la} presidencia de la Comunidad Autónoma

\begin{tabular}{|l|c|c|c|}
\hline $\begin{array}{c}\text { Comunidad } \\
\text { autónoma }\end{array}$ & $\begin{array}{c}\text { Gobierno } \\
\text { anterior }\end{array}$ & \multicolumn{1}{|c|}{$\begin{array}{c}\text { Gobierno } \\
\text { actual }\end{array}$} & $\begin{array}{c}\text { Aparecen referencias a la } \\
\text { radiotelevisón pública }\end{array}$ \\
\hline ANDALUCÍA & PSOE & $\begin{array}{l}\text { PSOE (Apoyado por } \\
\text { Ciudadanos) }\end{array}$ & $\mathrm{NO}$ \\
\hline ARAGÓN & PAC & PSOE (Apoyado por Podemos) & $\mathrm{SI}$ \\
\hline ASTURIAS & PPOE (Apoyado por IU) & $\mathrm{SI}$ \\
\hline BALEARES & CC & PSOE (Apoyado por Podemos) & $\mathrm{SI}$ \\
\hline CANARIAS & PP & PSOE (Apoyado por Podemos) & $\mathrm{NO}$ \\
\hline $\begin{array}{l}\text { CASTILLA-LA } \\
\text { MANCHA }\end{array}$ & CIU & JXSÍ (Apoyado por CUP) & $\mathrm{SI}$ \\
\hline CATALUÑA & PP & PSOE (Apoyado por Podemos) & $\mathrm{SI}$ \\
\hline EXTREMADURA & PP & PP (Apoyado por Ciudadanos) & $\mathrm{NO}$ \\
\hline MADRID & PP & PP (Apoyado por Ciudadanos) & $\mathrm{SI}$ \\
\hline MURCIA & PP & $\begin{array}{l}\text { PSOE (Apoyado por } \\
\text { Compromis/Podemos) }\end{array}$ \\
\hline VALENCIA & &
\end{tabular}

Fuente: Elaboración propia

En los programas marco autonómicos el PSOE propone "fusionar y actualizar en una ley la normativa encadenada en el tiempo, desde 2006 hasta 2015, que regula la actividad de la Corporación RTVE". Podemos defiende desgubernamentalizar los medios públicos y reformar la ley general de 2010 para adecuarla a los estándares europeos.xiv

Sin embargo, hay algunas diferencias de matiz en algunas comunidades. El PSOE propone una reforma de la ley del tercer canal para el caso de las Islas Baleares a fin de garantizar una red territorial insular de IB3; en el caso de Cataluña proponen la derogación de la Ley de la Corporació Catalana de Mitjans Audiovisualsxv promulgada por CIU y PP y para la Comunidad de Madrid la promulgación de una nueva ley para el Ente Público Telemadrid así como la reapertura de RTVV aspecto en el que Ximo Puig insistió en su programa de investidura.xvi 
Podemos, además de proponer en general impulsar el tercer sector, es decir, los servicios de comunicación comunitarios, se pronuncia en Aragón por fortalecer la CARTV como "servicio público y agente social económico", de igual manera en Asturias con la RTPA, en Valencia creando una nueva RTVV no partidista y punta de lanza del sector", y en Madrid propone un plan urgente para recuperar RTVM. En Cataluña, potenciando "Ios medios de comunicación públicos bajo control democrático ciudadano". Es decir, hay una confluencia en temas como la reapertura de RTVV o una nueva ley para Telemadrid salvo por parte del PP. Y en los datos obtenidos de las entrevistas hay una clarísima apuesta por el servicio público de televisión.

\subsection{Coexistencia de la Televisión pública nacional y las autonómicas}

Los partidos políticos analizados apuestan por la coexistencia entre la televisión pública nacional y los entes autonómicos, aunque la relación entre RTVE y las televisiones autonómicas no aparece explícitamente en los programas electorales, los datos son los que obtuvimos del análisis de las entrevistas. Solo podemos encontrar referencias indirectas como la de Ciudadanos: "RTVE debe coordinar el encuentro con todas las televisiones autonómicas apoyando su viabilidad". xvii "

Este debate se pone de relieve a partir de la derogación de la ley del tercer canal, vigente desde el 83 , por parte de la ley de la comunicación audiovisual del 2010, de la posibilidad abierta a la privatización de los canales públicos referida por el Presidente Mariano Rajoy en su discurso de investidura del 2011: "Cuando las administraciones están abocadas a la reducción del gasto, es absolutamente prioritario revisar nuestro actual modelo de televisiones públicas. Por ello, les anuncio que, con carácter inmediato, traeremos a esta Cámara la reforma legal necesaria para permitir nuevos modelos de gestión."xviii Y por las afirmaciones en el mismo sentido de los portavoces de UTECA: "UTECA, la asociación de las televisiones nacionales comerciales, insiste en presentar como la mejor opción la privatización de las televisiones autonómicas y la renuncia de éstas a la publicidad" (Azurmendi, López y Manfredi (2011:1). Finalmente, la modificación de la ley de comunicación audiovisual en el 2012 abrió la puerta definitivamente a la posibilidad de liquidar o privatizar las cadenas públicas deficitarias, lo que ocurrió en Valencia al año siguiente.

Ciudadanos, PSOE y Podemos apuestan claramente por la coexistencia de los dos servicios públicos de televisión, haciendo especial hincapié en la protección de la diversidad lingüística y en la información de proximidad. El PP, matiza su apoyo señalando que son los responsables autonómicos los que deben de decidir la necesidad y sus objetivos. No cierra la puerta a la gestión privada de las televisiones autonómicas: "Creemos profundamente en la televisión pública nacional y por tanto consideramos que debe existir y coexistir con opciones privadas o comerciales y también con ofertas autonómicas (públicas o privadas)" (Entrevista PP).

A la pregunta de ¿̇Considera adecuada la coexistencia de centros territoriales de RTVE y de cadenas autonómicas? , los papeles se invierten, mientras que PSOE y Podemos hablan de cooperación, para evitar duplicidades de gasto e infraestructura y de un tamaño ponderado, el PP apuesta rotundamente por la existencia de los centros territoriales de RTVE "que es una de las grandes fortalezas de la Corporación, uno de sus activos más rentables y justificados y una de las actividades que más y mejor ayudan a apoyar y valorar la razón de ser de RTVE". (Entrevista PP).

\subsection{Gobernanza y transparencia}

PSOE, Ciudadanos y Podemos, defienden el regreso al modelo implantado por el gobierno de Zapatero en la ley de junio del 2006 de elección del Presidente a través de una mayoría reforzada de dos tercios y se pronuncian en contra del decreto de abril del 2012 que modificó el régimen administrativo de la Corporación de RTVE. Podemos propone además mejorar la ley del 2006 adaptándola a Europa con "el modelo de agencia independientes, como la BBC británica" y en el caso de Ciudadanos que el Presidente sea elegido por mayoría de dos tercios entre una terna elegida por concurso publico de candidatosxix. El Partido Popular es el único que mantiene el sistema actual.

También en los otros ámbitos territoriales los partidos políticos y coaliciones se plantean reformas legislativas que refuercen la profesionalización de los entes públicos. La coalición catalana de Junts pel sí sostiene, en su programa electoral, la necesidad de "Reforzar más la independencia gubernamental de la CCMA a través de reformas legislativas que profesionalicen la gobernanza, garantizando su financiación a través del contrato programa de 4 años, estabilizando el sistema mixto de financiación, así como adscribiendo su dependencia y control exclusivamente al Parlamento"..xx 
Tanto en el vaciado de los programas (generales y autonómicos) como en la entrevista estructurada a la que han respondido los partidos hemos indagado en otros aspectos concretos de la gobernanza: la figura del Consejo de Administración, sobre el que todos se pronuncian por la reforma de los actuales, su elección parlamentaria por mayoría de dos tercios, profesionalización, independencia y la reducción en su composición. Ciudadanos propone además que dé cabida a un amplio abanico de organizaciones sociales a través de puestos rotatorios.xi

Para el PP es necesaria una reducción de los miembros del Consejo de la Corporación de RTVE y apuntan a la modificación que su gobierno hizo en 2012: De 12 miembros a 9, supresión de las retribuciones, recursos humanos y técnicos que tenían desde la ley 2006. El PSOE respecto a la composición, forma de elección y retribuciones aboga por fórmulas garantistas de su independencia, y la proporcionalidad: los sueldos dependerán de sus funciones y estas de que los consejeros tengan dedicación exclusiva o dedicación parcial.

Podemos y sus confluencias es el partido que más concreta su modelo ya que a la elección del Presidente y del Consejo de Administración añade:

- Los representantes de los trabajadores en el Consejo de Administración se elegirán por votación directa específica como ocurre con el Consejo de Informativos.

- El referéndum acerca del Jefe de Informativos pasará a tener carácter vinculante.

- Elección del Defensor de la Audiencia por parte de la ciudadanía, en votación directa a través de medios interactivos. Formará parte del Consejo de Administración y rotará cada tres años.

- Se creará un Consejo Social amplio y con competencias a través del cual la sociedad civil pueda decidir los principios de gestión de la radiotelevisión pública y vigilar su cumplimiento.xxii

De los datos de las entrevistas se constata que los cuatro partidos políticos están de acuerdo en la reducción del número de directivos de las televisiones públicas y de sus sueldos. En el caso de las plantillas, Podemos propone la racionalización progresiva "para adaptarse a las estructuras productivas del siglo XXI, a partir de planes consensuados con la representación de los trabajadores". Mientras que para el PSOE no es necesaria la reducción sino una mejor gestión de la plantilla existente. Contradictoriamente, el PP defiende el mantenimiento de plantillas de la Corporación de RTVE, "un medio tan amplio en canales y actividades como RTVE debe disponer siempre de la plantilla más adecuada para sus funciones", mientras que administraciones autonómicas del Partido Popular promovieron grandes expedientes de regulación de empleo en los entes de Valencia o Madrid durante las pasadas legislaturas.

Todos los partidos están de acuerdo en la utilidad de las Comisiones de Control parlamentario tal y como está establecido, solo Podemos añade un matiz que es potenciar su función fiscalizadora. También coinciden PSOE, Podemos y Ciudadanos en la falta de transparencia en la gestión de las televisiones públicas y en sus mecanismos de contratación de programas. El PP, al contestar a la entrevista, se sitúa en el polo opuesto "En RTVE la transparencia, el control, el rigor y los mecanismos para el cumplimiento de las normas de contratación son completos y así lo detectan las últimas auditorías públicas realizadas".

Estos tres partidos también coinciden sin fisuras en la no externalización de los servicios informativos y en el caso de la entrevista a Podemos proponen el control ciudadano: "Ios informativos son un producto estratégico y sensible, cuya calidad debe garantizarse y auditarse a través de un Observatorio participativo que recoja el feedback aportado por los públicos, trabajadores, consejos de informativos, asociaciones profesionales, partidos y sociedad civil" (Entrevista Podemos).xaii

\subsection{Financiación y sostenibilidad del modelo de televisión pública}

Todos los partidos coinciden en la necesidad de mejorar el actual sistema de financiación de la Corporación de RTVE con nuevos ingresos que garanticen su continuidad y su independencia financiera. Ninguno de estos partidos, según sus programas electorales, está dispuesto a defender el regreso de la publicidad a TVE. Del mismo modo que todos los partidos, incluidos los nacionalistas como el PNV con respecto a EITB o la coalición Junts pel si para la Corporació Catalana de Mijans Audiovisuals, defienden igualmente un modelo de financiación mixta con publicidad para cada una de sus cadenas de ámbito territorial.xxiv

Concretamente, en este apartado, y del análisis de los programas electorales a las elecciones generales, el PP es el único que no se pronuncia. Ciudadanos propone reformar las tres grandes leyes que regulan la 
actividad de RTVE: Estatuto, Mandato Marco y sobre todo Ley de Financiación, para que garantice una financiación estable y suficientexxv. EI PSOE justifica la supresión de la publicidad en RTVE a partir de Ley 8/2009, de 28 de agosto, aunque propone mejorar el sistema de financiación de la Corporación RTVE con nuevos ingresos y con nuevos mecanismos que garanticen la continuidad, la suficiencia e independencia financiera de la Corporación.

En el caso de Podemos tampoco se concreta cómo ha de ser la financiación, aunque abogan para que exista un modelo de financiación estable y que permita una "Gestión eficaz y transparente. Para cortar de raíz con la opacidad y los alarmantes casos de corrupción, se adoptará un estricto código de transparencia e incompatibilidades. Toda la contabilidad será íntegramente publicada en una web específica accesible al contribuyentexxvi. Del análisis de los datos se observa que ningún partido concreta cómo ha de ser el futuro modelo de financiación de la televisión pública nacional, incluso el Partido Popular defiende alcanzar el mayor acuerdo posible para ello.

Del análisis de los programas autonómicos, es reseñable cómo el programa electoral del PSOE para la Comunidad de Aragón se pronuncia por el mantenimiento de un modelo mixto: financiación pública más publicidadxxvii.

A través de las contestaciones a la entrevista hemos conseguido concretar algo más por lo que se refiere a la financiación de las televisiones públicas. Para el PP "la financiación de RTVE es un elemento esencial que hay que reconsiderar a medio plazo y a la luz de sus más que evidentes disfunciones. La materia merece alcanzar el mayor acuerdo sin cerrar ninguna puerta inicialmente (salvo la del canon)" Considera el PP que la actual financiación de RTVE es suficiente, aunque debe estar abierta a los retos que se puedan plantear en el futuro.

El PSOE justifica el actual sistema de financiación:

Si se hacen las cuentas la supresión de la publicidad en la CRTVE y el nuevo sistema de la tasa a las "telecos" -pese a problemas administrativos y legales- es un método más rentable. Totalmente justificado. La ley debe ir en esa línea, pero mejorada, para evitar pleitos y problemas de interpretación o aplicación. El sistema de patrocinios debería también clarificarse más. (Entrevista PSOExxviii).

Podemos mantiene igualmente una financiación sin publicidad para TVE y una financiación mixta con publicidad a nivel general, pues apunta en la entrevista alguna excepción "en aquellas televisiones públicas donde el funcionamiento sin publicidad directa es ya un sello de identidad del servicio público". La financiación actual les parece suficiente, siempre y cuando se racionalice el gasto de "los salarios astronómicos de algunos directivos y de reducir la estructura salarial de pirámide invertida".

Ciudadanos no cree que la solución sea la vuelta a la publicidad, pero tampoco comparte la ley de 2009 de modo que la financiación de RTVE quede a expensas de las cuentas de otras empresas:

Se ha de revisar claramente el modelo de financiación de RTVE, pero siendo muy conscientes de que la vuelta de la publicidad en absoluto compensaría los ingresos derivados de los impuestos establecidos tras la reforma de 2009. Se ha de apostar por una financiación estable, en modo alguno dependiente de las cuentas de otras empresas y de los vaivenes de los gobiernos de turno. (Entrevista Ciudadanos). $\times$ xix

Todos los partidos son conscientes de la imposibilidad de implantar un canon, por lo que lo excluyen de la posible financiación, aunque en el caso de Podemos con matices:

primero sería necesario rehabilitar la reputación de las televisiones de titularidad, para que el contribuyente la sienta suya y la perciba como una herramienta útil, como ocurre en otros países de Europa. Si esto no ocurre, no es una propuesta razonable. (Entrevista Podemos).

Respecto al futuro y la sostenibilidad del modelo, todos los partidos unánimemente ven la viabilidad de sus respectivos entes públicos de radiotelevisión como los motores del sector audiovisual de su respectivo ámbito territorial. Ésta, junto con la información de proximidad, es una de las razones de ser de las televisiones públicas autonómicas.

Aun así Podemos ve amenazada la sostenibilidad de las televisiones públicas por la caída de audiencias y de la reputación social perdida de algunos entes autonómicos, por ello las medidas para asegurar la sostenibilidad en el futuro irían encaminadas a:

recuperar la misión de servicio público (cercanía, pluralismo, rigor, participación) además de potenciar la transparencia para racionalizar el gasto al máximo, y buscar mayores audiencias para que los patrocinios y espacios publicitarios mejoren su aportación. Se debe permitir la explotación 
comercial de la producción propia, así como de los archivos audiovisuales de titularidad pública. (Entrevista Podemos).

Mayor es la ambigüedad del PSOE quien considera que "Es necesario una redefinición del modelo" o del PP "no hay otra medida más que asegurar su rentabilidad social", sin hacer mayor concreción de la financiación y viabilidad de las televisiones públicas.

\subsection{Consejo Audiovisual}

Todos los partidos políticos, excepto el PP, son partidarios de la creación de un órgano regulador del audiovisual homologable al modelo europeo, y todos ellos se pronuncian en contra del sistema actual de la Comisión Nacional de los Mercados y la Competencia (CNMC). La creación de un consejo audiovisual independiente (CEMA) estaba previsto en la ley $2010 x x$ y tras quedar pendiente su creación en la última legislatura socialista, el gobierno del Partido Popular se ha pronunciado reiteradamente en contra hasta que finalmente incluyó las funciones que la ley 2010 atribuía al CEMA en la CNMC como (Gavara 2012).

Incluso Ciudadanos, que se había pronunciado en las elecciones catalanas, junto con el PP por suprimir el Consejo Audiovisual de Cataluña (CAC), "porque lejos de ser un órgano regulador es un órgano político cuya misión es sancionar a los medios que no comulgan con la ideología nacionalista", también se muestra partidario de la creación de un regulador convergente, audiovisual/ telecomunicaciones, de ámbito nacional que trabaje de manera coordinada con los reguladores audiovisuales de las comunidades autónomas. (Entrevista Ciudadanos).

El PSOE y Podemos sí abordan este tema en sus programas con detalle. En el caso de Podemos abogan por la creación de un Consejo Audiovisual independiente "integrado en la plataforma Europea de Autoridades Reguladoras (EPRA), similar a los que existen en la mayoría de los países europeos"xaxi El PSOE define su finalidad:

garantizar la supervisión, la transparencia y el pluralismo en el convergente sector audiovisual y el de las telecomunicaciones, así como el cumplimiento de la misión y las obligaciones legales atribuidas a los prestadores del servicio público de comunicación audiovisual. [...]Constitución efectiva del Consejo Estatal de Medios Audiovisuales, que contribuya a la protección de los pequeños proyectos empresariales o comunitarios, así como una regulación de los conflictos de interés. Y como consecuencia de la concentración de los grandes grupos (Programa PSOE: 133).xxii

En la entrevista también se preguntó por los Consejos audiovisuales tanto a nivel nacional como autonómico y las respuestas fueron parecidas. El PP reitera que:

no hace falta crear nuevos organismos y estructuras de control si ya hay otros que llevan a cabo estas funciones. Recordemos que RTVE, por ejemplo, está sometida al control del Consejo de Administración del Consejo Asesor, del Parlamento, de la IGAE, del Tribunal de Cuentas, de los auditores privados externos, de los auditores internos, de la CNMC... No parece que haya mucho margen para nuevas figuras de control sin chocar con las que ya existen (Entrevista PP).

Ciudadanos aboga por un regulador a nivel nacional y reguladores autonómicos que trabajen coordinados. Concretamente, su portavoz, señala en la entrevista que:

La Comisión Nacional de los Mercados y de la Competencia (CNMC) es un macrorregulador que se aleja por completo de los modelos del entorno europeo. Se han de separar claramente lo que debería ser un regulador convergente (audiovisual y telecomunicaciones) y la autoridad de la Competencia.

Se ha de garantizar la independencia de este regulador convergente y dotarlo de más competencias, en particular la adjudicación de licencias de cobertura estatal.

El regulador convergente de ámbito nacional debería trabajar de manera coordinada con los reguladores audiovisuales de las comunidades autónomas. Uno y otros deberían ampliar su ámbito de actuación a los nuevos medios digitales (Entrevista Ciudadanos).

Podemos también defiende su existencia en el mismo sentido en el que se manifiesta en su programa electoral, reclama la existencia de los consejos en las Comunidades Autónomas, aunque abogando por "la racionalización de estructuras para el ahorro de costes, tomando como ejemplo el modelo alemán, donde los consejos regionales se coordinan a nivel nacional para cooperar, compartir recursos y unificar criterio". Al ser preguntados sobre una posible modificación de los ya existentes proponen un paso más en el sentido de la participación ciudadana: 
sería deseable que afronten la necesidad de crear Observatorios Ciudadanos para medir el retorno social de la inversión en medios públicos, en base a los indicadores publicados por la UNESCO y/o los que se utilizan en este sentido en otras televisiones europeas como la BBC o la ZDF, además de garantizar la participación social en la gestión y elaboración de contenidos (Derecho Constitucional de Acceso) para lo que es imprescindible fomentar la transparencia integral del sistema de medios (Entrevista Podemos).

\section{Discusión y conclusiones}

1. Lo primero que se observa del análisis general, tanto de los programas electorales para las elecciones autonómicas como para las generales como del resto de materiales analizados, es que la tradicional dicotomía o polarización entre las opciones de izquierda política y de derecha para los medios de comunicación públicos ya no son tan decisivas como hace años. En principio tanto los cuatro partidos políticos principales como el resto de formaciones o coaliciones se muestran partidarios pública y unánimemente de la preservación de los servicios públicos de radiotelevisión en nuestro país de cara al futuro. Incluso, el Partido Popular se pronuncia a favor de "la libertad, la independencia y la objetividad de los medios públicos de comunicación".

2. Este análisis se planteó como hipótesis inicial de trabajo que, a raíz de los nuevos movimientos de contestación social y de la presencia subsiguiente de nuevos partidos a partir del 2011, se había recuperado el debate sobre el futuro de la televisión pública. En segundo lugar, al no contar ningún partido con mayorías absolutas en las consultas electorales analizadas, el escenario y contexto político para la televisión pública se había hecho más favorable al reparto de poder, por lo que forma parte de los elementos de consenso y negociación entre unas fuerzas políticas y otras. En tercer lugar, que al encontrarse en minoría respecto al general apoyo a la radiotelevisión pública, el Partido Popular, -partido cuyo comportamiento o experiencia de gestión, tanto en administración nacional como en administración autonómica, ha cuestionado más su viabilidad- parece haber evolucionado a favor respecto a este servicio público, al menos en sus declaraciones públicas. Y parece ahora, en contraste con los hechos acaecidos en los últimos años, que todas las formaciones políticas buscan mejores fórmulas para asegurar su viabilidad futura, como por ejemplo ha sucedido con la recuperación de la televisión valenciana o con la nueva ley de Telemadrid, Murcia o Baleares.

3. En resumen, el futuro de la televisión pública en España se ha introducido en la agenda de las formaciones políticas. La segmentación del panorama político español y la desaparición de las mayorías absolutas ha propiciado el protagonismo del debate sobre el servicio público de la televisión, incluso ha sido elemento clave en las negociaciones para las investiduras de algunos nuevos presidentes como por ejemplo en las comunidades Valencia, Madrid, Aragón, Murcia, (cf. Cuadro 1). Hasta puede afirmarse que la fortaleza y viabilidad del servicio público de televisión es ahora mayor que, por ejemplo, en 2012 cuando existía otro escenario en el parlamento nacional o en varias comunidades autónomas.

4. Desde 1980 (Ley 4/1980, de 10 de enero, de Estatuto de la Radio y la Televisión) el futuro del servicio público de radiotelevisión estatal dependía en su fórmula de gestión del partido del gobierno de turno. La Ley 17/2006 de 5 de junio, en el gobierno de Rodríguez Zapatero modificó este aspecto al encomendar a la Cámara por mayoría de dos tercios la elección de su presidente por un mandato de 6 años; en la siguiente legislatura de Mariano Rajoy de nuevo se modificó este aspecto (Real Decreto 15/2012 de 20 de abril), pero aunque sigue vigente este marco legal, en la actualidad los cuatro partidos políticos principales, y el resto de formaciones o coaliciones analizadas, se muestran partidarios de la preservación de los servicios públicos de televisión, lo que resulta ya una novedad destacable que se ve reflejada en las propuestas y declaraciones para las elecciones autonómicas y generales. Aspecto este que no se había producido en anteriores comicios.

5. El PP es el que tiene una posición más diferenciada y que en ocasiones llega a la contradicción respecto a las radiotelevisiones autonómicas, sobre todo en aquellas comunidades que no están gobernadas por ellos ya que en unos casos apoya su carácter público (Andalucía), y en otros se muestra partidario de su privatización (Canarias). En nuestra entrevista, este partido contestó posicionándose claramente a favor de la titularidad pública de la radiotelevisión nacional y de las autonómicas, aunque concretara que "existen fórmulas en algunas autonomías de titularidad pública y gestión privada muy útiles". La posición del PP parece haber evolucionado como consecuencia del cambio de escenario político y de no haber dado resultado su experiencia de cierre de alguna cadena, por lo que ahora optarían por la externalización o gestión privada en aquellos casos que la sostenibilidad de la cadena se pusiera en cuestión. También puede haber contribuido el posicionamiento de Ciudadanos a favor de la imparcialidad y autonomía de los entes autonómicos, y de la despolitización y la transparencia en la CRTVE. 
6. Aunque la relación entre la CRTVE y las televisiones públicas autonómicas no aparezca explícitamente en los programas electorales, según los partidos políticos analizados está garantizada la coexistencia futura entre la televisión pública nacional y las autonómicas. Ciudadanos, PSOE y Podemos apuestan claramente por esa coexistencia de los dos servicios públicos, haciendo especial hincapié en la protección de la diversidad lingüística y en la información de proximidad. El PP, matiza su apoyo señalando a los responsables autonómicos como los que deben de decidir la necesidad y sus objetivos.

7. Todos los partidos políticos excepto el PP tienen objetivos bastante similares en cuanto a la gobernanza y la transparencia de los medios públicos, pero mientras que el PP es el único que defiende el modelo actual, PSOE, Podemos y Ciudadanos son favorables a retomar la elección del presidente por mayoría parlamentaria reforzada de dos tercios, y por dotar de mayor transparencia la gestión y las contrataciones de contenidos y por la no externalización de los informativos.

8. Todos los partidos coinciden en la necesidad de mejorar el actual sistema de financiación de la CRTVE con nuevos ingresos que garanticen su continuidad y su independencia financiera. Pero ninguno de estos partidos está dispuesto a defender el regreso de la publicidad a TVE. Del mismo modo que todos los partidos, incluidos los nacionalistas como el PNV con respecto a EITB o la coalición Junts pel si para la Corporació Catalana de Mijans Audiovisuals, defienden igualmente un modelo de financiación mixta con publicidad para cada una de sus cadenas de ámbito territorial. Por lo que es previsible que se mantenga en el futuro el modelo de financiación mixta para las empresas autonómicas.

9. Todos los partidos políticos, excepto el PP, son partidarios de la creación de un órgano regulador del audiovisual homologable al modelo europeo, y todos ellos se pronuncian en contra del sistema actual de la CNMC. Ciudadanos, que se había pronunciado en las elecciones catalanas, junto con el PP, por suprimir el Consejo Audiovisual de Cataluña (CAC), también se muestra partidario de la creación de un regulador convergente, audiovisual/ telecomunicaciones, de ámbito nacional que trabaje de manera coordinada con los reguladores audiovisuales de las comunidades autónomas. Podemos reclama la existencia de los consejos en las Comunidades Autónomas, aunque abogando por "la racionalización de estructuras para el ahorro de costes, tomando como ejemplo el modelo alemán, donde los consejos regionales se coordinan a nivel nacional para cooperar, compartir recursos y unificar criterio" y proponen un paso más en el sentido de la participación ciudadana:

10. Por todo ello también podemos concluir que el nuevo contexto político que ha resultado de las consultas electorales analizadas otorgan un mayor papel al sistema o modelo profesionalizado y autónomo respecto a la futura regulación de la radiotelevisión pública, como todos los partidos políticos han enunciado en sus programas y declaraciones. Para definir el concepto de profesionalización, lo hacemos como sinónimo de objetividad o neutralidad política, basándonos en las tres dimensiones de Hallín y Mancini (2004:31):

- Ejercicio de la autonomía profesional.

- Respeto a las normativas profesionales específicas.

- Orientación hacia el servicio público de los contenidos.

11. Además de existir en los pronunciamientos de los partidos políticos un mayor grado de unanimidad, podemos deducir que el sistema de radiotelevisión pública tiene hoy mayor y mejor futuro que hace pocos años, puesto que los propios partidos políticos, incluido el Partido Popular, proponen un gran acuerdo para establecer el futuro modelo de financiación. El futuro de la televisión pública ya no está en manos de la alternancia de dos partidos con visiones contrapuestas, sino que han entrado en el debate abierto por nuevas formaciones políticas que incorporan también nuevas visiones y nuevas generaciones sociales. Frente a la alternancia de los dos principales partidos en el gobierno, con sus visiones y concepciones contrapuestas, la sostenibilidad de los medios públicos de radiotelevisión autonómica y nacional, estaba menos garantizada que con el nuevo escenario multipartidista. La aparición de nuevos actores políticos, resultado del nuevo escenario del proceso electoral, ha puesto la atención en mayor medida sobre la necesidad de asegurar la viabilidad de los medios públicos. Por ello ha reforzado la necesidad de mantener el futuro de los medios públicos desde una gestión que garantice la profesionalidad y la independencia, como bases para reforzar su credibilidad social.

\section{Referencias ${ }^{\mathrm{x} x \mathrm{xii}}$}

[1] Azurmendi, A.; López, N. y Manfredi, J.L. (2011). La reforma de la televisión pública autonómica en el nuevo marco legal audiovisual (Ley 7/2010, General de la Comunicación Audiovisual), Derecom, (5), 124. 
[2] Bustamante, E. (2010). La comunicación en las comunidades autónomas. Un déficit democrático fuerte, Informe sobre la Democracia. Madrid: Fundación Alternativas.

[3] Bustamante, E. (2013a). Historia de la radio y la televisión en España. Barcelona: Gedisa.

[4] Bustamante, E. (2013b). La comunicación social en España: profunda regresión democrática del pluralismo y la diversidad. Gaceta sindical: reflexión y debate, Madrid: CCOO, (21), 233-252.

[5] Caffarel, C. (2007): El servicio público de televisión en España, Journal of Spanish Cultural Studies, Special Issue: New Approachees to Spanish Television, Routledge, Taylor \& Francis, 85-94.

[6] Caffarel, C. y García de Castro, M. (2006): Editorial autonomy and public control, the debate over reform. In C.S. Nissen (ed.). Making a Differenc. Public Service Brodcasting in the European Media Landscape, (pp. 135-146). UK: John Libbey Publishing.

[7] Campos Freire, F. (2013). El futuro de la TV europea es híbrido, convergente y cada vez menos público. Revista Latina de Comunicación Social, (68), 89-118. doi:10.4185/RLCS-2013-970.

[8] De Mateo, R. y Bergés, L. (2009). Los retos de las televisiones públicas. Financiación, servicio público y libre mercado. Sevilla: Editorial Comunicación Social.

[9] FORTA (2012). Informe sobre el papel de la televisión pública autonómica en España. Madrid: FORTAACCENTURE. Disponible en goo.gl/qLmOzT

[10] Gavara de Cara, J.C. (2012). El CEMA como autoridad independiente de control de los medios de comunicación audiovisual y su reconversión en la CNMC como autoridad integrada y multisectorial del mercado y la competencia. En Gavara de Cara (ed.). Las autoridades independientes de control de los medios de comunicación audiovisual, (pp. 23-52). Barcelona: Bosch.

[11] García de Catro, M. (2006). ¿Para qué sirven los medios públicos? La reforma de la televisión pública. TELOS: Cuadernos de comunicación e innovación, (67),105-114.

[12] ] García de Catro, M. (2006). La autonomía de la televisión pública. Revista de Occidente, (302303), 160-177.

[13] Hallin, D.C. y Mancini, P. (2004). Comparing Media Systems: Three Models of Media and Politics. Cambridge: Cambridge University Press.

[14] Herrero Díaz, O. y Reyes Sánchez, F. (2016). Los efectos profesionales de la crisis de la televisión pública española: el creador audiovisual low cost. Ad Comunica. Revista Científica de Estrategias, Tendencias e Innovación en Comunicación, (1 1), 95-1 18. doi:10.6035/2174-0992.2016.11.7

[15] Jivkova-Semova, D. (2011). RTVE sin publicidad: un modelo de financiación en estado de emergencia, Vivat Academia, Año XIV (116), 75-91. doi:10.15178/va.201 1.116.75-91.

[16] Ley 2/2012, de 1 de agosto, de modificación de la Ley $7 / 2010$, de 31 de marzo, General de la Comunicación Audiovisual para flexibilizar los modos de gestión de los servicios públicos de comunicación audiovisual autonómicos. Disponible en goo.gl/5Efjtk

[17] Ley Orgánica 2/2012, de 27 de abril, de Estabilidad Presupuestaria y Sostenibilidad Financiera. Disponible en goo.gl/J3sl3j

[18] Ley 7/2010, de 31 de marzo, General de la Comunicación Audiovisual. Disponible en goo.gl/r7mgli

[19] Ley $17 / 2006$, de 5 de junio, de la radio y la televisión de titularidad estatal. Disponible en goo.gl/6XbBdW

[20] López Cepeda, A.M. (2012). Modelos audiovisuales públicos en España. Perfil profesional, empresarial y político de sus principales órganos de gestión, Comunicación y Sociedad, 25 (1), 399-427.

[21] López Jordán, J. (2013). ¿̇Son necesarias las televisiones públicas en España? Un debate adulterado. adComunica. Revista Científica de Estrategias, Tendencias e Innovación en Comunicación, (6), 233-237.

[22] López Rico, C.M. (2016). La reapertura de radio televisión valenciana. De la manipulación al servicio público. Revista Mediterránea de Comunicación 7(2), 243-257. doi:10.14198/MEDCOM2016.7.2.15

[23] Lowe, G.F. y Bardoel, J. (2007). (Eds.). (2007): From Public Service Broadcasting to Public Service Media. Göteborg, Sweden: Nordicom.

[24] Marzal Felici, J. (ed.) (2015): Las televisiones públicas autonómicas del siglo XXI. Barcelona: UAB, Aldea Global, 34. 
[25] Marzal, J.; Izquierdo, J. y Casero, A. (2015). La crisis de la televisión pública. El caso de RTVV y los retos de una nueva gobernanza. Castellón: UJI, UAB, UPF, UV.

[26] Miguel de Bustos, J.C. y Casado del Río, M.A. (2015). La crisis de la radiotelevisión pública autonómica en Marzal, J., Izquierdo, J. \& Casero, A. (Eds.), La crisis de la televisión pública. (pp. 61-78). Castellón: UJI, UAB, UPF, UV.

[27] Moreno, E. y Jiménez, E. (Eds.) (2007) Los desafíos de la televisión pública en Europa. Navarra: EUNSA.

[28] Ortiz Sobrino, M.A. (2010). El nuevo marco legal para la financiación de la radio televisión pública estatal en España. Comunicación y hombre, 6, 257-271. Disponible en goo.gl/9BvdlR

[29] Zallo, R. (2010). La política de Comunicación Audiovisual del gobierno socialista (2004-2009): un giro neoliberal, Revista Latina de Comunicación Social, 65, 14-29. Disponible en goo.gl/By2k4C

\footnotetext{
i Este trabajo de investigación ha sido financiado por la Cátedra Unesco de investigación en Comunicación y por la Asociación de periodistas europeos. En el trabajo participaron también los profesores de la URJC, Juan Francisco Torregrosa y Yolanda Ortiz de Guinea.

"Los resultados de la primera fase de la investigación, el que concernía a las televisiones autonómicas fue presentado como ponencia en el Congreso del AICE celebrado en Madrid en julio pasado.

iii Las elecciones se celebraron en 15 de las 17 comunidades autónomas ya que País Vasco y Galicia las celebrarán en 2016.

iv Mantenemos el orden alfabético de los partidos y no el orden por número de escaños ya que al referirnos también a comicios autonómicos el resultado no es homogéneo.

$\vee$ En Inglaterra la BBC, en Francia, France Televisión o en Italia la RAI se encargan de las desconexiones territoriales sin que existan televisiones regionales públicas parecidas a nuestra FORTA, por el contrario, en Alemania hay dos sistemas de televisión pública la regional ARD3 que cuenta con 9 canales regionales que operan en igual número de regiones y a nivel nacional ARD1 y ZDF que no tienen desconexiones regionales. En España, como caso particular, nos encontramos con las televisiones públicas autonómicas (regionales) y con CRTVE que también se encarga de producir desconexiones territoriales.
}

vi La muestra de periódicos seleccionados para el seguimiento de las campañas electorales ha sido el resultado de elegir, para cada comunidad autónoma, el periódico de mayor difusión según los datos del EGM y en el ámbito de las elecciones generales los dos periódicos de mayor difusión El País y El Mundo también según los datos del EGM del periodo electoral analizado.. Disponible en goo.gl/TeWbY5

vii goo.gl/UrkvfD (pp. 132-136).

viii goo.gl/7ihvSq (pp. 51-52)

${ }^{i x}$ goo.gl/pqPZRF (pp.251-253)

× goo.gl/WgJHZc (p.53) Hay que tener en cuenta que el Estatuto de Autonomía Andaluz blinda el carácter público de la RTVA.

${ }^{x i}$ goo.gl/ThPxGG (p.24)

xii Ramón Moreno Bustos, es el portavoz del PP en la Comisión mixta de control parlamentario sobre RTVE. La entrevista fue grabada el día 11/03/2016 por Juan Francisco Torregrosa

xiii Programa autonómico de ciudadanos (C's) para Castilla la Mancha. pdf. Candidato Ángel Ligero López. (p.54) El programa y el resto de programas de Ciudadanos en las elecciones autonómicas han sido eliminados en la web desde que se convocaron las elecciones generales de 2016

xiv https://goo.gl/J6cLrG (p.134). goo.gl/Wr5Yce

xv Ley 11/2007, de 11 de octubre, de la Corporación Catalana de Medios Audiovisuales, BOPC 136/08.

xvi https://goo.gl/yTMVIF

xvii goo.gl/DHGZOY (p.252) Queremos aclarar que Ciudadanos ha retirado todos sus programas electorales de la red antes de las elecciones de junio de 2016, por lo que los tenemos que citar en webs indirectas El PDF es el mismo que el original.

xviii p. 20 del Discurso de investidura de Mariano Rajoy, disponible en goo.gl/MCO8ju

xix goo.gl/DHGZoY (p.252).

${ }^{x x} \mathrm{goo} . \mathrm{gl} / \mathrm{KD} 2 \mathrm{xNp}(\mathrm{p} .116)$ 
wi goo.gl/P2FWgA (p.252)

xai goo.gl/DgUx13 (p.53)

xiii Miguel Álvarez Peralta es el coordinador de políticas mediáticas de Podemos. La entrevista fue grabada el 12 de enero de 2016 por Mario García de Castro

xiv goo.gl//rl7J15 (p.116)

xav goo.gl/DHGZOY (p.253)

xavi goo.gl/DgUxl3 (p.53)

xavi goo.gl//Hyis9 (p.177)

xaviii Miguel Angel Sacaluga es responsable del PSOE y representante por ese partido en el Consejo de administración de RTVE. La entrevista fue grabada el 10de marzo de 2016 por el Dr.Juan Francisco Torregrosa

xix Marta Martín Llaguno es diputada por Ciudadanos, catedrática de Comunicación y responsable de Medios de Comunicación. La entrevista fue grabada el 7 de abril de 2016 por Mario García de Castro

wa Ley 7/2010, de 31 de marzo, General de la Comunicación Audiovisual, BOE» núm. 79, de 01/04/2010

${ }^{x \infty i}$ goo.gl/Syhyfc (p.54)

waxi goo.gl/UrkvfD (p.133)

waxii Las Webs correspondientes a los programas electorales consultados (generales 2015 y 2016, autonómicas marco y específicas de las comunidades Autónomas 2015) han sido reseñadas como notas a pié de página 